$\xi=-1$

\title{
Forecasting Electricity Consumption Using Time Series Model
}

\author{
Y.W. Lee' ${ }^{1}$ K.G. Tay ${ }^{2 *}$, Y.Y. Choy ${ }^{3}$ \\ ${ }^{1}$ Department of Power Engineering, Faculty of Electrical and Electronic Engineering, Universiti Tun Hussein Onn Malaysia \\ ${ }^{2}$ Department of Communication Engineering, Faculty of Electrical and Electronic Engineering, Universiti Tun Hussein Onn Malaysia \\ ${ }^{3}$ Department of Mathematics, Faculty of Applied Science and Technology, Universiti Tun Hussein Onn Malaysia \\ *Corresponding author E-mail: tay@uthm.edu.my
}

\begin{abstract}
Electricity demand forecasting is important for planning and facility expansion in the electricity sector. Accurate forecasts can save operating and maintenance costs, increased the reliability of power supply and delivery system, and correct decisions for future development. Universiti Tun Hussein Onn Malaysia (UTHM) which is a developing university in Malaysia has been growing since its formation in 1993. Thus, it is important for UTHM to forecast the electricity consumption in future so that the future development can be determined. Hence, UTHM electricity consumption was forecasted by using the simple moving average (SMA), weighted moving average (WMA), simple exponential smoothing (SES), Holt linear trend (HL), Holt-Winters (HW) and centered moving average (CMA). The monthly electricity consumption from January 2011 to December 2017 was used to forecast January to December 2018 monthly electricity consumption. HW gives the smallest mean absolute error (MAE) and mean absolute percentage error (MAPE), while CMA produces the lowest mean square error (MSE) and root mean square error (RMSE). As there is a decreasing population of UTHM after the moving of four faculties to Pagoh and HW forecasted trend is decreasing whereas CMA is increasing, hence HW might forecast better in this problem.
\end{abstract}

Keywords: Centered Moving Average; Holt Linear Trend; Holt-Winters; MAE; MAPE; MSE; RMSE; Simple Exponential Smoothing; Simple Moving Average; Weighted Moving Average.

\section{Introduction}

Time series is a collection of equally spaced temporal data. A time series can consist some or all of the components such as trend (long term pattern), cyclical (repeated up and down movements), seasonal (regular fluctuations during the same month or quarter) and irregular (unexplained random component) [1].

Forecasting is predicting future values based on past and current time series data. Forecasting for future load demand is essential for future power system planning and control. Load forecasting can be divided into short-term load forecasting (STLF), mediumterm load forecasting (MTLF) and long-term load forecasting (LTLF). STLF up to one day or one week at most, MTF ranges from one day to several months while LTF forecasts more than a year ahead) [2]. STLF is used for scheduling the generation and transmission of electricity, MTLF is used to plan the fuel purchases, whereas LTLS is aimed to develop the power supply and delivery system (generation units, transmission system, and distribution system) [3].

Universiti Tun Hussein Onn Malaysia (UTHM) is one of the Malaysian technical university located in Parit Raja, Batu Pahat, Johor Malaysia. It was formerly known as Pusat Latihan Staf Politeknik (PLSP) which was established in 1993. There is a great development of UTHM infrastructure after UTHM has been upgraded from Institut Teknologi Tun Hussein Onn (ITTHO) in 1996, Kolej Universiti Teknologi Tun Hussein Onn (KUiTTHO) in July 2001 to UTHM in Jan 2007.

There are four new buildings for Faculty of Technical and Vocational Education (FPTV), Faculty of Technology Management \& Business (FPTP), Faculty of Civil and Environmental Engineering
(FKAAS), Faculty of Computer Science and Information Technology (FSKTM) and several new buildings (Complex of Faculty of Electronic and Engineering (FKEE) and Faculty of Mechanical and Manufacturing (FKMP), as well as new library, were built. However, in August 2017, three faculties of UTHM which is Faculty of Applied Science and Technology (FAST), Faculty of Technology Engineering and Diploma Studies Centre were moved to Pagoh, Johor. On top of that, there is a new building of FKEE which is completed in 2018.

The development of new buildings and movement of the above three faculties to Pagoh will affect the electricity demand. Hence, there is a need to forecast the UTHM electricity consumption for future decisions on generating electric power, load switching, and infrastructure development. As the nature of our data is time series, so the most widely used time series forecasting models in this field will be adopted to forecast UTHM electricity consumption.

There are various forecasting methods available in the literature such as multiple linear regression, time series model, Auto regressive integrated moving average (ARIMA) process, artificial neural network, Fuzzy time series, Fuzzy neural network, and etc. In this paper, the related time series model reviews will be carried on only as we chose to forecast UTHM electricity consumption by time series model. Tsokos [4] implemented $k-$ th moving average, a $k$ - th weighted moving average and $k-$ th exponential moving average to formulate the best ARIMA model to forecast a real stock price data from the Fortune 500 list.

Ostertagova and Ostertag [5] used simple exponential smoothing (SES) on number of personnel in the industrial production in Slovakia over the years $2001-2010$. Meanwhile, Ostertagova and Ostertag [6] utilized SES on primary production of electricity in terajoules in Slovakia over the year 2001-2009. The accuracy of 
the SES depends on the value of smoothing factor, $\alpha$. They determined the optimal value of $\alpha$ used traditional optimization method based on the lowest mean absolute error (MAE), mean absolute percentage error (MAPE) and root mean square error (RMSE).

Abd Jalil, Ahmad and Mohamed [7] applied Holt-Winters (HW), Holt-Winters for double multiplicative seasonality proposed by Taylor (HWT) and two variations of HWT on a half-hourly load demand of Malaysia from September 01, 2005 to August 31, 2006. They used the 11 months of data to estimate methods parameters and the remaining 1 month to evaluate post-sample forecasting performance. They concluded that HWT performed better than HW.

Nazim and Afthanorhan [8] compared SES, Double Exponential Smoothing (DES), Holt's (Brown) and Adaptive Response Rate Exponential Smoothing (ARRES) techniques on the secondary data of Malaysia population covering the period 1957 up to 2013. Holt's method was found to be the best method to forecast the Malaysia population as produces the lowest Mean Square Error (MSE) value

Muhamad and Mohamad Din [9] employed single and double exponential smoothing as well as Holt's method on river water level data from three rivers in Perlis Malaysia. They found that double exponential smoothing technique was the best in this problem.

Kavanagh [10] employed double seasonal exponential smoothing variations of Holt-Winters method on STLF. He obtained an average daily MAPE of $2.99 \%$ over a period of nearly four weeks.

Tirkeş, Güray and Çelebi [11] examined the performances between Trend Analysis, Decomposition and HW models on monthly time series data by a group of jam and sherbet product demands from January 2013 to December 2014. HW and Decomposition models obtained better results in their study.

Setiawan, Juniati and Farida [12] implemented triple exponential; smoothing methods (Winter) with a variation of alpha, beta and gamma on number passenger of Kereta Api Indonesia Ltd (PT KAI) Bandung Indonesia for Argo Wilis, Turangga, Mutiara Selatan, Pasundan and Kahuripan trains between 2006 and 2014.

Popeanga and Lungu [1] adopted centered moving average method and time series analysis on quartely 2010-2013 final energy consumption in Romania. They forecasted 2014 final energy consumption and the method showed promising results.

Therefore, in this paper, six time series methods were applied to the monthly UTHM electricity consumption from January 2011 to December 2017 to forecast the UTHM monthly 2018 electricity consumption. The accuracy of each method will be compared.

\section{Time Series Analysis Methods}

Here, six time series analysis methods will be utilized in forecasting UTHM electricity consumption which are simple moving average, weighted moving average, simple exponential shooting, Holt linear trend, Holt-Winters and deseasonalization. The monthly UTHM electricity consumption data from January 2011 to December 2017 was obtained from Department of Property and Development, UTHM.

\subsection{The Simple Moving Average (SMA $(m))$}

The easiest time series method is averaging the given time series to get an overall idea of the trends and to smooth the time series. The simple moving average (SMA $(m)$ ) of $m$ number of data from a time series consists of $n$ number of data is the average values of $m$ of the previous values defined by

$$
\hat{y}_{t}=\frac{y_{t-m}+y_{t-m+1}+\cdots+y_{t-1}}{m}, \quad m+1 \leq t \leq n
$$

where $\hat{y}_{t}$ represents the forecasted load at time $t, y_{t}$ is real load at time $t$. In this study, the SMA of three months was taken, hence $m$ was taken as 3 and we get $\operatorname{SMA}(3)$. Thus, $\hat{y}_{4}$ is actually the average value of $y_{1}, y_{2}, y_{3}$. In general, $\hat{y}_{t}$ is the average value of previous $n$ - month data.

\subsection{Weighted Moving Average (WMA)}

SMA gives the same weight for each dataset, but weighted moving average (WMA) has different weight for each dataset as follows:

$\hat{y}_{t}=\frac{\mathrm{w}_{1} y_{t-m}+\mathrm{w}_{2} y_{t-m+1}+\cdots+w_{m} y_{t-1}}{m}, \quad m+1 \leq t \leq n$

Where $w_{t}$ is the weight for the corresponding data determined by minimizing sum of square of error (SSE). Initially, the values of $w_{1}, w_{2}, w_{3}$ are randomly set, then their correct values will be found by minimizing SSE using Solver in Excel.

\subsection{Simple Exponential Smoothing (SES)}

The Simple Exponential Smoothing weights recent data more than previous one [13]. SES smooths the time series like SMA and uses the smoothed series in forecasting future values [6]. It can be represented by the following equations:

$$
\begin{aligned}
& \hat{y}_{1}=y_{1} \\
& \hat{y}_{t+1}=\alpha y_{t}+(1-\alpha) \hat{y}_{t}
\end{aligned}
$$

where $\alpha$ is the smoothing constant between 0 and 1 which determines the accuracy of forecasting. The value of $\alpha$ was initially randomly set in its range then its optimal value will be obtained by setting the minimum value of MSE in Solver in EXCEL. SES is suitable if a time series fluctuates or moves randomly above and below about a constant level or stationary series [13], has no trend and no seasonal patterns [14].

\subsection{Holt Linear Trend (HLT)}

Holt's Linear Trend (HLT) method is a double exponential shooting which adds the second exponential smoothing parameter, $v_{t}$ to smooth the trend of the time series data. It can be used when there is a linear trend in the time series. The equation of Holt's Linear Trend is as follows:

$u_{1}=y_{1}$

$v_{1}=0$

$u_{t}=\alpha y_{t}+(1-\alpha)\left(u_{t-1}+v_{t-1}\right)$

$v_{t}=\beta\left(u_{t}-u_{t-1}\right)+(1-\beta) v_{t-1}$

$\hat{y}_{t+1}=u_{t}+v_{t}$

Here $u_{t}$ and $v_{t}$ are the smoothed constant process value and smoothed trend value respectively. $\alpha$ denotes smoothing factor, while $\beta$ is smoothing parameter for the trend. Both values of $\alpha$ and $\beta$ must be equal to or less than 1 . Same as in WMA and SES methods, the optimized values of $\alpha$ and $\beta$ can be found by minimizing the SSE using Solver in Excel. 


\subsection{Holt-Winters (HW)}

Holt-Winters (HW) method is triple exponential smoothing which adds in the seasonal component $s_{t}$ if compared to Holts Linear Trend method. It can be used if the time series is seasonal [10]. The Holt-Winters method is written as:

The initial values of seasonal component, $s_{t}$ which $1 \leq t \leq c$, is given as below:

$$
s_{t}=\frac{y_{t}}{\frac{1}{c} \sum_{i=1}^{c} y_{t}}
$$

Here $c$ is the length of a seasonal circle. $c=12$ as there are 12 months in a year. Next

$$
\begin{aligned}
& u_{c}=\frac{y_{c}}{s_{c}} \\
& v_{c}=0
\end{aligned}
$$

For $t>c$, the following formula will be used

$$
\begin{aligned}
& u_{t}=\alpha\left(\frac{y_{t}}{s_{t-c}}\right)+(1-\alpha)\left(u_{t-1}+v_{t-1}\right), \\
& v_{t}=\beta\left(u_{t}-u_{t-1}\right)+(1-\beta) v_{t-1}, \\
& s_{t}=\gamma\left(\frac{y_{t}}{u_{t}}\right)+(1-\gamma) s_{t-c}, \\
& \hat{y}_{t}=\left(u_{t-1}+v_{t-1}\right) s_{t-c} .
\end{aligned}
$$

Similar with the concept of Holt's Linear Trend method, the values of $\alpha, \beta$ and $\gamma$ were randomly set which $\alpha$ and $\beta$ must be equal or less than 1 while the sum of $\alpha$ and $\gamma$ must be less than 1 . Then the optimized values of $\alpha, \beta$ and $\gamma$ were found by minimizing the SSE using Solver in Excel.

\subsection{Centered Moving Average (CMA $(m))$}

If a time series exhibits regular seasonal fluctuations then for the purposes of analysis (for example, to estimate an underlying trend) it is often necessary to remove the seasonal fluctuations to leave deseasonalized data. To use deseasonalized data trend to forecast we proceed with the SMA of 12 months, SMA(12) equation defined as:

$$
u_{t}=\frac{y_{t-\frac{m}{2}}+y_{t-\frac{m}{2}+1}+\cdots y_{t+\frac{m}{2}-1}}{m}, \frac{m}{2}+1 \leq t \leq n-\left(\frac{m}{2}-1\right)
$$

Here $m=12$ as we take $\operatorname{SMA}(12)$.

Later, the center moving average $\mathrm{CMA}(12)$ is found by averaging the two SMA(12) as follows:

$v_{t}=\frac{u_{t}+u_{t+1}}{2}, \frac{m}{2}+1 \leq t \leq n-\left(\frac{m}{2}-1\right)$

The specific seasonal for each month is computed by dividing actual data by $\mathrm{CMA}(12)$ as

$s_{t}=\frac{y_{t}}{v_{t}}, \frac{m}{2}+1 \leq t \leq n-\left(\frac{m}{2}-1\right)$

Next, the seasonal index for each month $k$ is calculated as the average of specific seasonal for month $k$ for all years as follows:
$S_{t(k)}=\frac{S_{2011, k}+S_{2012, k}+\ldots+S_{2017, k}}{\frac{m}{2}}, \quad 1 \leq t \leq n, \quad k=1,2, \ldots 12$

Then to remove the effect of seasonal variation, the actual data for each month is divided by the seasonal index for that month as

$D_{t}=\frac{y_{t}}{s_{t(k)}}, \quad 1 \leq t \leq n$

where $D_{t}$ is deseasonalized index. Later, by using simple linear regression (SLR) on time $t$ as the independent variable and $D_{t}$ as the dependent variable, the trend was obtained as

$T_{t}=a+b t, \quad 1 \leq t \leq n$

Where $a$ is the intercept and $b$ is the coefficient of the SLR. Both the values of $a$ and $b$ can be found by using the Regression function from Data Analysis option from Data menu in Excel.

To install the Data Analysis ToolPak in Microsoft Excel just click 'File' at the menu bar $>>$ select 'Options' $>>$ click 'Add-Ins' $>>$ click 'Analysis ToolPak' $>>$ click 'Go...' >> tick the 'Analysis ToolPak' at the 'Add-Ins' table $>>$ click 'Ok'.

Click 'Data' menu $>$ click 'Data Analysis' $>>$ choose 'Regression' >> click 'Ok'.

Selecting $D_{t}$ as 'Input Y Range' while $t$ as 'Input $X$ Range'. Then check on the 'Labels' if include the label and Confidence Levels checkboxes. Next, click the 'Output Range' and type in any desired cell in EXCEL. Finally, click 'Ok'.

Lastly, the forecasted load is

$\hat{y}_{t}=s_{t(k)} T_{t}, \quad 1 \leq t \leq n$

\section{Error analysis}

The performance of the above time series methods can be measured by mean absolute error (MAE), mean absolute percentage error, mean square error (MSE), root mean square error (RMSE) as below:

$M A E=\frac{\sum_{t=1}^{n}\left|y_{t}-\hat{y}_{t}\right|}{n}$

$M A P E=\frac{\sum_{t=1}^{n} \frac{\left|y_{t}-\hat{y}_{t}\right|}{y_{t}}}{n} \times 100 \%$,

$M S E=\frac{\sum_{t=1}^{n}\left|y_{t}-\hat{\mathrm{y}}_{t}\right|^{2}}{n}$,

$R M S E=\sqrt{\frac{\sum_{t=1}^{n}\left|y_{t}-\hat{y}_{t}\right|^{2}}{n}}$,

where $y_{t}, \hat{\mathrm{y}}_{t}$ are real and forecasted data respectively, $n$ is the number of real data. Here, MAE measures the average value of the absolute error or the average of the spread of error. All errors are assigned equal weights in MAE [6]. MAPE is relative percentage error corresponds to MAE. Lewis [15] stressed that the MAPE is the most useful measure to compare the accuracy of forecasting methods as it measures relative performance. A MAPE which is less than 10 percent is considered as highly accurate forecasting, between 10 - 20 percent is good forecasting, between $20-50$ percent is interpreted as reasonable forecasting and over 50 percent is inaccurate forecasting [15]. On the other hand, MSE measures the average of the squares of the errors, 
hence large errors are given additional weight [6]. Whereas RMSE is the square root of the MSE.

\section{Results and Discussions}

UTHM electricity consumption patterns versus month for years 2011-2018 is shown in Figure 1. It is noticed that the electricity consumption has increased since the year 2011. The electricity consumption fluctuates for each month. The electricity consumption in December 2017 is the minimum if compared to the same December month as 3 faculties of UTHM moved to Pagoh since Aug 2017. The year 2015 has the highest electricity consumption of $3869.05 \mathrm{MWh}$, while the year 2011 has the minimum electricity consumption if compared to other years. The electricity consumption for certain months are low than usual may because the month is mostly semester break of UTHM. There are fewer students in the campus and therefore, the electricity consumption will be less if compared to the months that are not semester break.

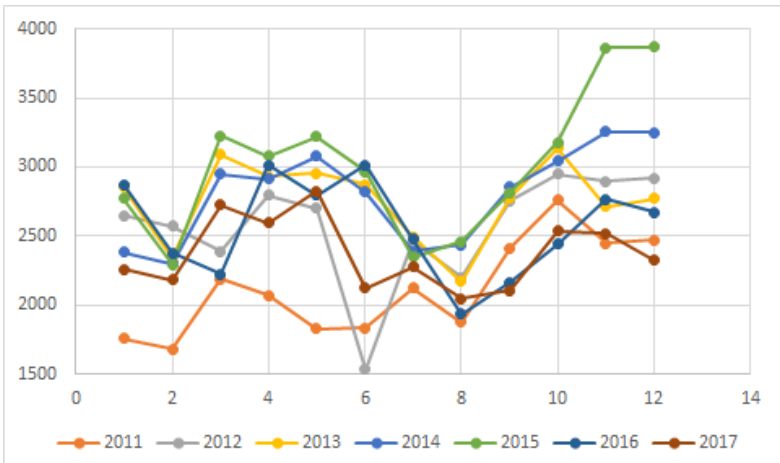

Fig. 1: Actual Load by years

Figure 2 shows the time series of UTHM electricity consumption continuously from Jan 2011-Dec 2017. The electricity consumption is range from $1500 \mathrm{MWh}$ to $4000 \mathrm{MWh}$. The time series seems is not stationary and is increasing. The electricity consumption in the year 2018 will be forecasted by using six different time series analysis methods.

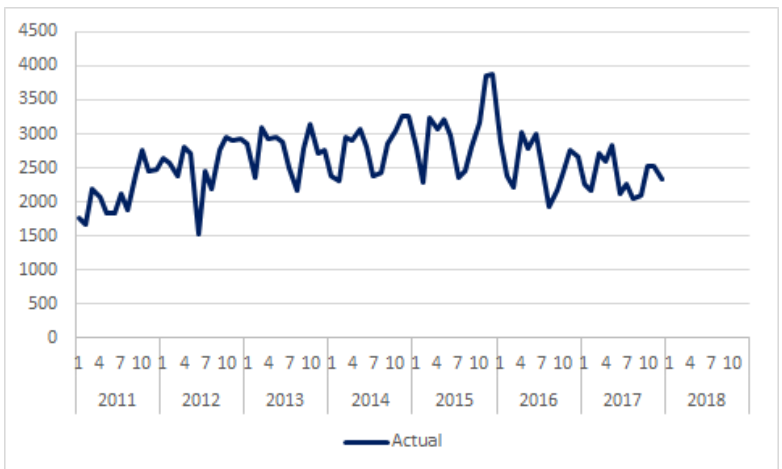

Fig. 2: Actual Electricity Consumption for all years

The actual UTHM electricity consumption (blue colour) from Jan 2011 to December 2017 and forecasted electricity consumption (red colour) using MA(3) from April 2011 to December 2018 were depicted in Figure 3. MA(3) uses the average of previous three-month electricity consumptions to forecast the fourth-month electricity consumption. Hence, the forecasted results start in April 2011. MA(3) can forecast the pattern of the actual data but the pattern is shifted to the right. The future forecasted pattern of $\mathrm{MA}(3)$ is linear.

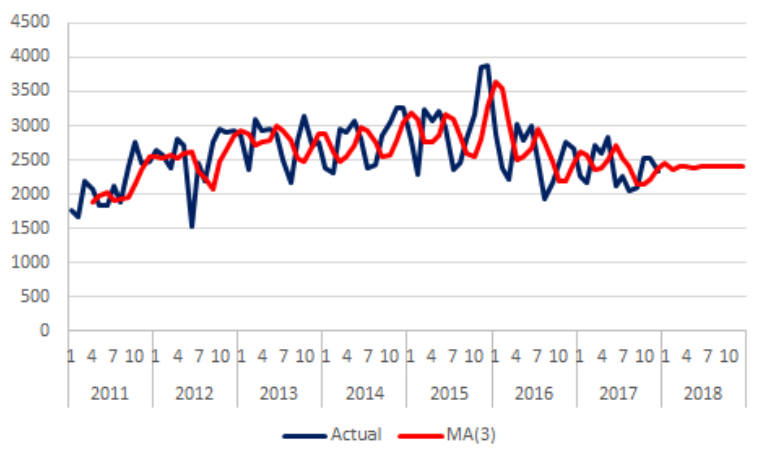

Fig. 3: Actual Electricity Consumption and MA(3)

By using WMA with $w_{1}=0.1897, w_{2}=-0.0336$ and $w_{3}=0.8439$, its forecasted results is shown in red colour line in Figure 4. Figure 4 shows that WMA can forecast quite closely to the actual data if compared to $\mathrm{MA}(3)$, but its future forecasted pattern is linear follows MA(3).

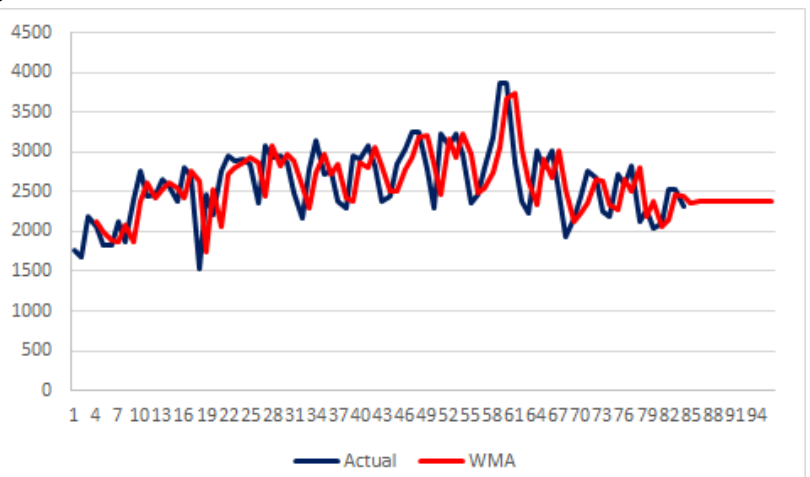

Fig. 4: Actual Electricity Consumption and WMA

The forecasted results by using SES with $\alpha=0.2857$ is displayed in red colour line in Figure 5. SES forecasted pattern is poorer if compared to $\mathrm{MA}(3)$, but its future forecasted pattern is linear follows MA(3).

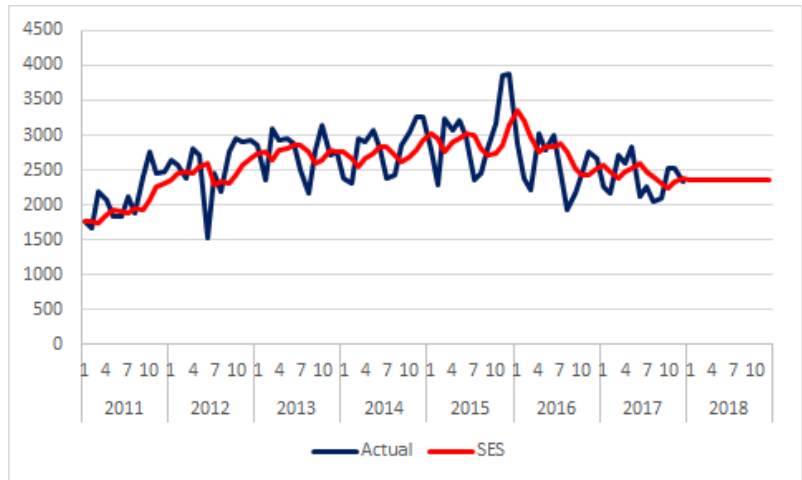

Fig. 5: Actual Electricity Consumption and SES

HLT with $\alpha=0.2921$ and $\beta=-0.005$ were plotted in Figure 6 . In HLT, the forecasted values are slightly reducing which shows the trend of the data. The future forecasted pattern of HLT is linear too. 


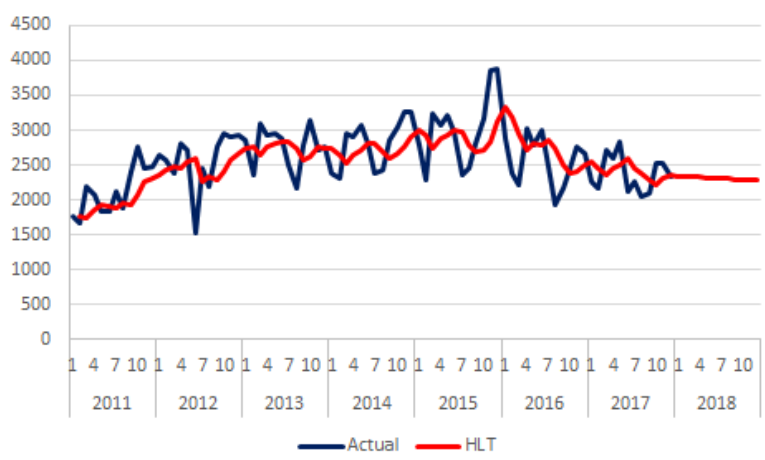

Fig. 6: Actual Electricity Consumption and HLT

HW with $\alpha=0.1631 \beta=-0.0168$ and $\gamma=0.4433$ were depicted in Figure 7. HW used previous 12-month electricity consumptions to forecast the thirteen-month electricity consumption. Thus, the forecasted results start from Jan 2012. The seasonality from the previous data was used to forecast the electricity consumption for the year 2018. Thus, the forecasted values may more accurate. HW future forecasted pattern is no longer linear if compared to the previous fourth methods but the future trend is decreasing.

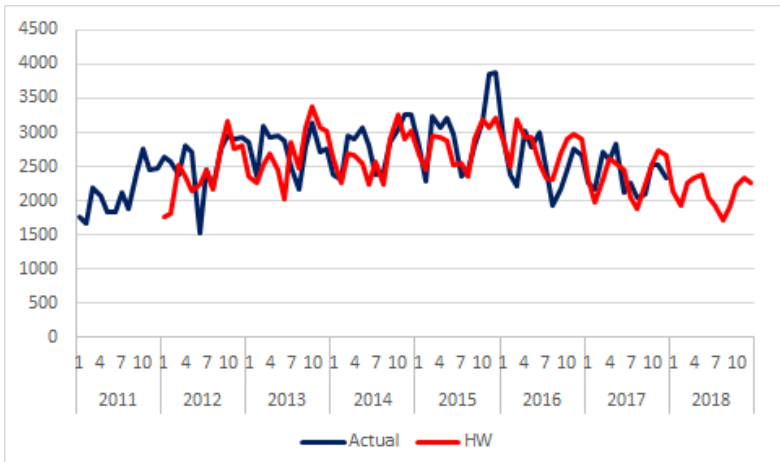

Fig. 7: Actual Electricity Consumption and HW

Figure 8 displays the forecasted electricity consumption by using CMA. It can forecast from Jan 2011 to December 2018 follows the pattern but their values are lower than the actual value. Similarly, to HW, the future forecasted pattern is nonlinear but increasing.

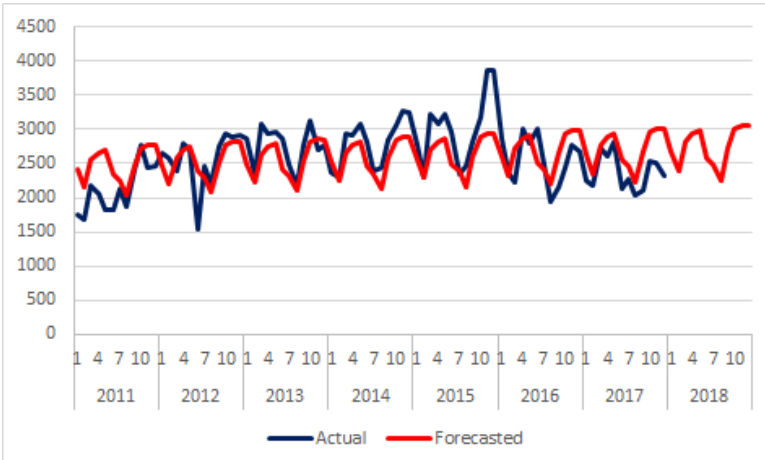

Fig. 8: Actual Electricity Consumption and Centered Moving Average

Table 1 presents the forecast evaluation in terms of MAE, MAPE, MSE and RMSE values for SMA, WMA, SES, HL, HW and CMA methods. HW gives the lowest MAE (293.2291) and MAPE(11.14\%), while CMA gives the lowest MSE(130442.4) and RMSE(361.1681). On the other hand, SMA gives the highest MAE (367.645), MAPE(14.46\%), MSE (197586.5) and RMSE(444.5071). MAE measures the average deviation of the predicted to the actual data and it is in linear order, while RMSE measures the square root of the average of squared differences between prediction and actual observation. Since the errors are squared before they are averaged, the RMSE gives a relatively high weight to large errors. This means the RMSE should be more useful when large errors are particularly undesirable. Hence, it is very difficult to conclude if HW or CMA is better if using MAE, MAPE, MSE and RMSE simultaneously. If using MAE or MAPE, HW will be the best, if using MSE or RMSE, CMA will be the best model. However based on Lewis [15], MAPE is the most useful measure to compare the accuracy of forecasting methods as it measures relative performance, hence HW should be the best methodf in this case as it produces the smallest MAPE.

Table 1: Forecast Evaluation

\begin{tabular}{|c|c|c|c|c|}
\hline & MAE & MAPE(\%) & MSE & RMSE \\
\hline SMA & 367.6459 & 14.46 & 197586.5 & 444.5071 \\
\hline WMA & 313.2387 & 12.36 & 154249.4 & 392.746 \\
\hline SES & 314.0011 & 12.47 & 148824.4 & 375.7777 \\
\hline HLT & 319.4969 & 12.60 & 150267.1 & 387.6431 \\
\hline HW & 293.2291 & 11.14 & 136499.3 & 369.4581 \\
\hline CMA & 293.3757 & 11.88 & 130442.4 & 361.1681 \\
\hline
\end{tabular}

\section{Conclusion}

SMA, WMA, SES, HL, HW and CMA were applied on monthly UTHM electricity consumption from Jan 2011-December 2017 to forecast monthly 2018 UTHM electricity consumption. CMA gives the lowest MSE and RMSE, while HW yields the lowest MAE and MAPE. According to the decreasing of the population in UTHM after the moving of four faculties to Pagoh, HW might be more accurate as its future trends is decreasing if compared to CMA which is in increasing trend. Therefore, HW can be used to forecast future UTHM electricity consumption based on its lowest MAPE (11.14\%) and it is a good forecasting according to Lewis [15].

\section{Acknowledgement}

We would like to thank the UTHM Research Fund and UTHM Tier 12018 research grant vote number H258for financial support of this project.

\section{References}

[1] Popeanga J \& Lungu I, "Forecasting Final Energy Consumption using the Centered Moving Average Method and Time Series Analysis", Database Systems Journal, Vol.5, No. 1, (2014), pp. 4250.

[2] Pedregal DJ \& Trapero JR, "Mid-term hourly electricity forecasting based on a multi-rate approach", Energy Conversion and Management, Vol. 51, (2010), pp. 105-111.

[3] Almeshaiei E \& Soltan H, "A methodology for Electric Power Load Forecasting", Alexandria Engineering Journal, Vol. 50, (2011), pp. 137-144.

[4] Tsokos CP, "K-th Moving, Weighted and Exponential Moving Average for Time Series Forecasting Models", European Journal of Pure And Applied Mathematics, Vol. 3, No. 3, (2010), pp. 406-416.

[5] Ostertagová E \& Ostertag O, "The Simple Exponential Smoothing Model", Modelling of Mechanical and Mechatronic System International Conference, (2011), pp. 380-384.

[6] Ostertagová E \& Ostertag O. "Forecasting Using Simple Exponential Smoothing Method", Acta Electrotechnica et Informatica, Vol. 12, No. 3, (2012), pp. 62-66.

[7] Abd Jalil NA, Ahmad MH \& Mohamed N, "Electricity Load Demand Forecasting Using Exponential Smoothing Methods", World Applied Sciences Journal, Vol. 22, No. 11, (2013), pp. 1540-1543.

[8] Nazim A \& Afthanorhan A, "A comparison between single exponential smoothing (SES), double exponential smoothing (DES) holt's (brown) and adaptive response rate exponential smoothing (ARRES) techniques in forecasting Malaysia population", Global Journal of Mathematical Analysis, Vol. 2(, No. 4, (2014), pp. 276280. 
[9] Muhamad NS, \& Mohamed Din A, "Exponential Smoothing Techniques on Time Series River Water Level Data", Proceedings of the $5^{\text {th }}$ International Conference on Computing and Informatics, (2015), pp. 644-649.

[10] Kavanagh K, "Short Term Demand Forecasting for the Integrated Electricity Market”, Vol. 2, No. 1, (2017), pp.1-10.

[11] Tirkeş G, Güray C, \& Çelebi N, "Demand Forecasting: a Comparison Between the Holt-Winters, Trend Analysis and Decomposition Models", Tehnički Vjesnik, Vol. 24, No.2, (2017), pp. 503-509.

[12] Setiawan W, Juniati E, \& Farida I, "The use of Triple Exponential Smoothing Method (Winter) in Forecasting Passenger of PT Kereta Api Indonesia with Optimization Alpha, Beta, and Gamma Parameters", Proceeding - 2016 2nd International Conference on Science in Information Technology, ICSITech 2016: Information Science for Green Society and Environment, (2017), 198-202.

[13] Li ZP, Yu H, Liu, YC \& Liu FQ, “An Improved Adaptive Exponential Smoothing Model for Short-term Travel Time Forecasting of Urban Arterial Street", Acta Automatica Sinica, Vol. 34, No.11, (2008), pp. 1404-1409.

[14] YorucuV, "The Analysis of Forecasting Performance by Using Time Series Data for Two Mediterranean Islands", Review of Social, Economic \& Business Studies, Vol. 2, (2003), pp. 175-196.

[15] Lewis C. D. Industrial and Business Forecasting Methods, London, Butterworths, 1982. 